International Journal of Retina (IJRETINA) 2020, Volume 03, Number 02.

P-ISSN. 2614-8684, E-ISSN.2614-8536

\title{
RETINAL PRACTICE DURING COVID-19 ERA
}

\author{
Dr. Anant Bhosale ${ }^{1}$
}

Associate Professor Dr. Chandra Balaratnasingam, PhD. ${ }^{1}$

${ }^{1}$ Lions Eye Institute, University of Western Australia, Perth, Australia

\author{
${ }^{*}$ Correspondence to: \\ Associate Professor Dr. Chandra \\ Balaratnasingam, PhD, \\ Lions Eye Institute, University of \\ Western Australia, Perth, \\ Australia, \\ balaratnasingam@gmail.com
}

COVID 19 has emerged as a public health threat around the world and has transformed health care system globally. It has certainly brought in numerous unprecedented challenges that we have no experience to tackle with. Tremendous disruptions in daily life noted and have forced everyone to modify the way of Life. Ophthalmology and particularly Retina practice is no immune to this global malady. Particularly when Ophthalmology being considered non urgent speciality, moreover for patients the fear of contracting the viral infection grew and stay-at-home orders were implemented, many patients found themselves in a conundrum: Do I try to see my doctor now or wait until the peak comes down???

For Ophthalmologists, we have to device a way to practice and coexist with ongoing effect of COVID -19 and formulate protocols to be followed for safe practice.

Five mandatory measures in any Ophthalmology clinic $^{1}$

1. Screen for fever at the point-of-entry and elicit a history of travel to affected areas during the incubation period

2. Those positive for \#1, with no ophthalmic emergency, should be triaged to a designated medical facility.

3. Those positive for \#1 with an ophthalmic emergency should be seen by the staff geared in personal protective equipment

4. Patients with conjunctivitis, with or without \#1, should be seen by the staff geared in personal protective equipment in an isolated designated examination room with an isolated waiting area.

5. Barrier care including N95 masks/face shields for all physically close ophthalmic procedures and universal precautions with decontamination of applanation prisms, contact gonioscopes, laser contact lenses, B-scan, and ultrasonic biomicroscope probes, etc

Telemedicine and Artificial intelligence could be used as effective tools for triaging and primary assessment of retina patients especially staging of retinopathy without physical presence of a patient in a retina clinic $^{2}$. Chatbot is an effective way of triaging patients and automatically answer most of the routine questions of patients ${ }^{3}$.

On arrival to the hospital waiting area in the clinic, should have adequate spacing and chairs spaced out to maintain standard physical distancing as per WHO guidelines.

Home dilatation, by electronic prescriptions is an effective way to reduce waiting time of patient in the hospital and minimize staff-patient contact. This method would be more suitable for previously registered patients to retina clinic. In a situation where patient needs dilatation in eye clinic, no touch technique with patient retracting lower lid and instillation of drops should be practised. Alcohol sanitation of hands prior to slit lamp and indirect ophthalmoscopy can prevent cross contamination Slit lamps, OCT, laser machines should have a breath shield and lenses e.g. 90D can be covered with plastic cling film as an additional protection. Contact lens exam, scleral depression during Indirect Ophthalmoscopy should be avoided if possible. Minimal or no talking during fundus examination is advisable and patient can be guided gaze for exam with examiners hands. 
Wide angle imaging systems of retina can be used as treatment guide with reduced patient time. Optical Coherence tomography and OCT angiography are effective alternatives to reduce invasive tests like Fluorescein and Indocyanin angiography which involve closer physical contact and longer chair time. B-scan, if warranted, the probe should undergo cleaning precautions as per the guidelines as it comes in direct contact with patient skin. Electrophysiological tests can be avoided unless absolutely essential. Retinopathy of prematurity screening can be effectively done with telemedicine tools. Babies with ROP in Zone 1 should have low threshold of treatment and early treatment can reduce number of visits to the hospital. Treating babies with Antivegf/laser on the same day of diagnosis is effective.

Intravitreal injections are the most common practised procedure in retina clinic and needs meticulous care as per guidelines ${ }^{4}$ to prevent transmission of COVID. Prioritization of patients needing injections is crucial. Patients with Emergent needs of injection e.g ROP requiring anti-VEGF, Neovascular glaucoma, AMD, PCV can be treated the same day. Patients with stable vision especially minimal diabetic macular edema, stable vein occlusions can be treated and extended as maximum as possible to reduce hospital visits. During injections patient should wear a mask .Treating retina specialist, and assistant nurse should use all aseptic precautions along with N95 masks, PPE kits face shield. Minimal or no conversation in the injection/procedure room helps in lowering droplet spread. Longer acting medications e.g intravitreal steroids maybe preferred wherever possible to reduce number of hospital visits.

Retinal lasers can be performed with non-contact delivery systems e.g LIO wherever possible. Multispot laser can reduce chair time of patient.0.5\% hypoclorite solution can be used to clean $20 \mathrm{D}$ lens after use for laser indirect system.

Vitreoretinal surgery if indicated in a patient should undergo screening regarding fever, history of travel and contact with a person with symptoms for 2 weeks.COVID testing is advisable in patients undergoing surgeries involving mucosal contact e.g Scleral buckling. In patients tested positive for COVID -19 and needing emergency surgery experienced surgeons can be preferred than trainees to minimize surgical time .During the surgery, the surgeon should not enter the OT until the patient is fully stabilized on the anaesthesia circuit and the surgical team is adequately protected. ${ }^{5}$ Patients being operated should wear a surgical mask if being operated under local anaesthesia. Complete PPE kit with N95 masks for the surgeon and assistant minimises spread. Use of Povidone iodine is recommended as it is virucidal and disinfects the ocular surface and conjunctival cul-de-sac in 15 seconds $^{6}$. Noncontact surgical viewing systems are preferable and usage of viscoelastics to keep the eye wet instead of continuous irrigation is advisable. Proper surgical draping is important as a watertight sealing drape prevents aerosol dispersion from nose and mouth. Valved cannulas system during vitrectomy reduces fluid spills. If non valved cannulas are used, reducing intraocular pressure whenever possible prevents sudden egress of fluids. Diathermy is a known source of aerosolization ${ }^{7}$ and can be substituted with more precise method of using endolaser with enhanced duration o to cauterize bleeders. Active extrusion versus passive egress of fluid during fluid air exchange can prevent bubbling and reduce aerosolization. Minimal instrument exchanges are preferred to reduce turbulence in the eye and fluid activity at the port area.

Doctors in training are suffering from the stress of low patient load and deficit in conventional training. Innovative and effective methods of focussed training through webinars, online didactic lectures-case presentations, may be considered as means of imparting teaching and engaging students with high academic yield.

The authors wish to emphasize that this pandemic is a unique and dynamic situation. It is impossible to suggest something right or perfect for all situations and best practices may vary with time and according to region, state, and country. With ever evolving data and guidelines, it is of utmost importance to follow regular updates from the authorities and to act upon accordingly. With the suggestions put forth here, we believe that we are equipped to do just that in the COVID-19 era. ${ }^{8}$

To conclude, our way providing medical care are going to be different in the COVID-19 era. We have to make modifications in a way that suit our practice taking optimal care to provide efficient care to patients in a safe environment and prevent ocular morbidity as much as possible. 


\section{REFERENCES}

1. Khanna R, Honavar SG. All eyes on Coronavirus-What do we need to know as ophthalmologists. Indian J Ophthalmol 2020;68:549-53.

2. Saha SK, Fernando B, Cuadros J, Xiao D, Kanagasingam Y. Automated quality assessment of colour fundus images for diabetic retinopathy screening in telemedicine. J Digit Imaging 2018;31:869-78.

3. Bibault JE, Chaix B, GuillemasséA, Cousin S, EscandeA, Perrin M, et al. A chatbot versus physicians to provide information for patients with breast cancer: Blind, randomized controlled noninferiority trial. J Med Internet Res 2019;21:e15787

4. ASRSEmergentUrgentORprocedures.Availablefrom:https://www. asrs.org/content/documents/asrs-emergenturgentorprocedures. 1.pdf

5. Wong, J, Goh QY, Tan Z, Lie Sa, Tay YC, Ng SY, et al. Preparing for a COVID-19 pandemic: A review of operating room outbreak response measures in a large tertiary hospital in Singapore. [published online ahead of print $2020 \mathrm{Mar}$ 11] Can J Anesth 2020. doi: 10.1007/s12630-020-01620-9.

6. Eggers M, Eickmann M, Zorn J. Rapid and effective virucidal activity of povidone-iodine products against middle east respiratory syndrome coronavirus (MERS-CoV) and modified vaccinia virus Ankara (MVA). Infect Dis Ther 2015;4:491-501.

7. Coccolini F, Perrone G, Chiarugi M, Di Marzo F, Ansaloni L, Scandroglio I, et al. Surgery in COVID-19 patients: Operational directives. World J Emerg Surg 2020:25. doi: 10.1186/ s13017-020-00307-2

8. Parrish RK, Stewart MW, Duncan Powers SL. Ophthalmologists are more than eye doctors -in memoriam Li Wenliang. Am J Ophthalmol. 2020;213:A1-A 